\title{
Squeezing an Egg into a Worm: C. elegans Embryonic Morphogenesis
}

\author{
A.J. Piekny ${ }^{1,2}$ and P.E. Mains ${ }^{1, *}$ \\ ${ }^{1}$ Genes and Development Research Group, Department of Biochemistry and Molecular \\ Biology, University of Calgary, 3330 Hospital Dr. NW, Calgary, AB, Canada T2N 4N1; \\ ${ }^{2}$ Present address: Research Institute of Molecular Pathology, Dr. Bohr-Gasse 7, A-1030, \\ Vienna, Austria \\ E-mails: piekny@imp.univie.ac.at and mains@ucalgary.ca
}

Received May 22, 2003; Revised December 5, 2003; Accepted December 8, 2003; Published December 18, 2003

We review key morphogenetic events that occur during Caenorhabditis elegans (www.wormbase.orgl) embryogenesis. Morphogenesis transforms tissues from one shape into another through cell migrations and shape changes, often utilizing highly conserved actin-based contractile systems. Three major morphogenetic events occur during $C$. elegans embryogenesis: (1) dorsal intercalation, during which two rows of dorsal epidermal cells intercalate to form a single row; (2) ventral enclosure, where the dorsally located sheet of epidermal cells stretches to the ventral midline, encasing the embryo within a single epithelial sheet; and (3) elongation, during which actin-mediated contractions within the epithelial sheet lengthens the embryo. Here, we describe the known molecular players involved in each of these processes.

KEYWORDS: C. elegans, development, morphogenesis, actin, myosin, Rho-binding kinase

DOMAINS: genetics (worms), genetics (fly), cell biology, intercellular communication, development, intracellular communication, motility, embryology

\section{INTRODUCTION}

Morphogenetic events transform an embryo from one shape into another. These changes originate at the cellular level and are based primarily on cell migrations and alterations in cell shape. The first major rearrangement is gastrulation (not covered in this review), where mesodermal cells migrate into the embryo resulting in three germ layers (see "gastrulation" www.bio.unc.edu/faculty/goldstein/lab/movies.html to view this process in C. elegans). Other types of morphogenetic movements important for embryogenesis include epiboly, where a sheet of epithelial cells extends across an embryo, and purse-string contractions (analogous to wound healing), where shortening at the apical edge of a group of cells surrounding an open space draws the cells together to close the space[1,2,3,4,5,6]. Critical to these processes are rearrangements of the actin cytoskeleton signaled by the Rho family of small Ras-like GTPases (Rho, Rac, and CDC42)[7,8,9,10]. These molecules regulate actinbased structures including filopodia, lamellipodia, and focal adhesions, which are critical for cell shape 
changes, movements, and the contractile process. In addition, adherent cell junctions maintain epidermal cells in an epithelial sheet to ensure that migrations and shape changes occur in a coordinated manner.

The nematode C. elegans is ideal for studying morphogenesis at the cellular level because the worm is transparent throughout its life cycle and all cell divisions can be viewed in living animals by Nomarski microscopy[11,12]. The small size (1.2 mm adults), short generation time (3.5 days at $20^{\circ} \mathrm{C}$ ), large brood size (>300 progeny), and hermaphroditic sexual system facilitate genetic analysis of developmental processes[13,14]. Genetic approaches have a crucial advantage over cellular and biochemical approaches as they can identify genes independent of the gene product's abundance. Genetics can also reveal the existence of redundant pathways that are difficult to detect by other methods. The complete genomic sequence of $C$. elegans[15] coupled with transformation rescue of mutant phenotypes allows rapid positional cloning of genes. In addition, RNA interference (RNAi)[16] efficiently disrupts the expression of genes lacking known mutations, allowing rapid analysis of candidate genes implicated by other systems.

In this review we will describe embryonic morphogenesis, which transforms the C. elegans embryo from a roughly spherical shape into a tube-like, vermiform larva. This transformation occurs in the absence of cell proliferation (reviewed in [5,6], and see "normal development" at www.bio.unc.edu/faculty/goldstein/lab/movies.html) and is driven by cell migration and shape changes within the surface layer of embryonic epidermal cells (also referred to as the "hypodermis" in nematodes)[17,18,19,20]. Approximately $300 \mathrm{~min}$ postfertilization, the epidermal cells are found in three pairs of rows straddling the posterior dorsal midline of the embryonic surface (Fig. 1). These cells will undergo three major morphogenetic events. Initially, the two rows of epidermal cells immediately adjacent to the dorsal midline intercalate with one another[17]. As dorsal intercalation completes, the ventral-most rows of epidermal cells undergo epiboly and migrate to the ventral midline to enclose the embryo within an epithelium enclosure" www.bio.unc.edu/faculty/goldstein/lab/movies.html)[18]. On completion of ventral enclosure, an actinmediated contraction of the lateral row of epidermal cells causes these cells, and the embryo, to contract, resulting in a fourfold longitudinal lengthening of the embryo[19]. The vermiform shape is then maintained by secretion of a cuticle by the epidermal cells[20]. We will now discuss each of these morphogenetic events in terms of morphology and the regulatory molecules involved in each event (all genes that will be discussed are summarized in Table 1).

\section{DORSAL INTERCALATION}

Dorsal intercalation, the first major morphogenetic event occurs when the two rows of epidermal cells that flank the dorsal midline merge into a single row. These round cells become wedge shaped and extend basal processes (Figs. 1 and 2) that slide beneath the borders of neighboring cells toward the dorsal midline. Later, the cell bodies push their way into the new intercalated position to form a single row on the dorsal midline[17], and many of these cells fuse with one another.

Relatively few genes are known to be specifically involved in dorsal intercalation, possibly because the genes involved are essential for earlier morphogenetic events such as gastrulation (causing mutant embryos to die before displaying dorsal intercalation defects). In addition, redundant pathways may govern dorsal intercalation, precluding the identification of any single gene that is found in one or the other pathway.

die-1 (dorsal intercalation and elongation defective) encodes a C2H2 zinc finger transcriptional regulator required for proper dorsal intercalation[21]. In die-1 mutants, dorsal epidermal cells initiate intercalation properly, but the cell bodies fail to migrate. While ventral 


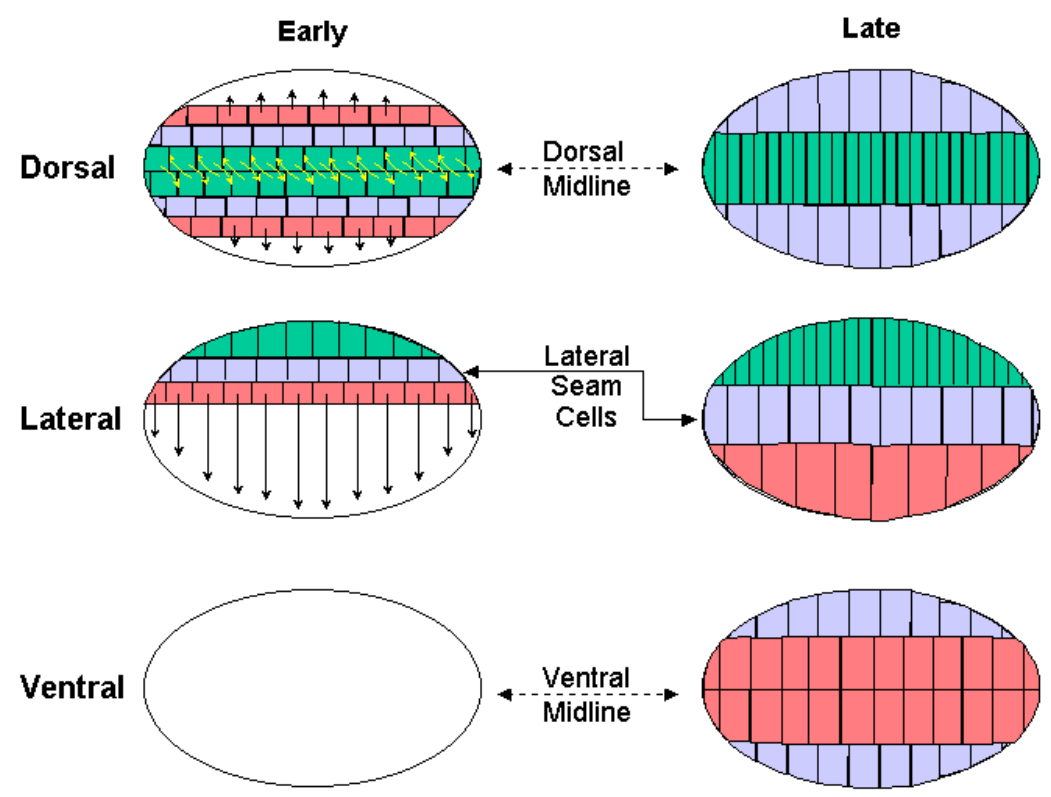

FIGURE 1. Schematic representation of the cell movements during dorsal intercalation and ventral enclosure. Initially, each side of the ventral midline contains three rows of epidermal cells. The dorsal-most rows (green) slide past one another as indicated by the arrows during dorsal intercalation, forming into a single row. Later, the ventral row (red) on either side of the dorsal midline migrates as indicated by the arrows to the ventral midline, enclosing the embryo.

enclosure is not defective, elongation fails. Pharynx, intestine, and muscle, which undergo embryonic morphogenetic changes similar to those in the epidermis, are also defective in die-1, suggesting that these morphogenetic events may utilize similar molecular mechanisms.

Intercalation movements fail in the C. elegans mutants of gex-2 (gut on the exterior) and gex-3: epidermal cells remain bunched on the dorsal surface of the embryo and ventral enclosure does not occur (see below)[22]. Dorsal intercalation similarly fails when wild-type embryos are treated with cytochalasin $\mathrm{D}$ (an inhibitor of actin polymerization), indicating that the actin cytoskeleton is essential for this process[19]. Indeed, the Arp2/3 (actin related protein) complex, a complex of seven polypeptides that nucleates actin polymerization in eukaryotes, is also required for intercalation movements and ventral enclosure in C. elegans[23]. In other eukaryotes, the protein WASP positively regulates actin polymerization via Arp2/3[24]. WASP contains protein motifs that bind various signaling molecules, such as Cdc42, a Rho-GTPase[24]. Perhaps related to this, the homologues of gex-2 and gex-3 interact with the Cdc42-related small GTPase RacA; RacA is a mediator of the actin-based lamellipodia required for cell migration in cultured fibroblast cells[10,22]. The protein products of the C. elegans genes, denoted GEX-2 and GEX-3, localize to cell boundaries and could mediate Rac signaling required for epidermal cell migration, although how they affect Rac is currently unknown[22].

\section{VENTRAL ENCLOSURE}

The next major morphogenetic event, ventral enclosure, begins prior to completion of dorsal intercalation ("ventral enclosure" at www.bio.unc.edu/faculty/goldstein/lab/movies.html). On each side of the embryo, the ventral and lateral rows of epidermal cells stretch and migrate to the ventral midline to enclose the embryo in a single continuous epithelial sheet whose integrity is critical for elongation of the embryo at a later stage (Figs. 1 and 2)[18]. Initially, two pairs of anterior ventral ("leading edge") cells extend filopodia and migrate ventrally over underlying 
TABLE 1

Genes Required for Morphogenesis

\begin{tabular}{|c|c|c|}
\hline Gene [Ref.] & Protein & Morphogenetic Event \\
\hline die-1 [21] & $\mathrm{C} 2 \mathrm{H} 2$ zinc finger transcription factor & Dorsal intercalation \\
\hline gex-2 [22] & Rac GTPase interacting protein & \\
\hline gex-3 [22] & Rac GTPase interacting protein & \\
\hline $\operatorname{arp} 2 / 3[23]$ & Microfilament nucleation & \\
\hline$v a b-1[25]$ & Ephrin receptor tyrosine kinase & Ventral enclosure \\
\hline efn $(1-4)[26,27]$ & Ephrin ligands & \\
\hline mab-20 [29] & Semaphorin-2a & \\
\hline ptp-3 [32] & LAR-like protein tyrosine phosphatase & \\
\hline $\operatorname{arp} 2 / 3[23]$ & Microfilament nucleation & \\
\hline gex-2 [22] & Rac GTPase interacting protein & \\
\hline gex-3 [22] & Rac GTPase interacting protein & \\
\hline ced-10 [33] & Rac GTPase & \\
\hline$h m r-1[35]$ & Cadherin & \\
\hline$h m p-1[35]$ & a-Catenin & \\
\hline$h m p-2$ [35] & $\beta$-Catenin & \\
\hline let-413 [45,49] & GTPase adaptor protein & Elongation \\
\hline$d l g-1[46,47,49]$ & MAGUK & \\
\hline ajm-1 [48] & Novel coiled coil protein & \\
\hline sma-1 [50] & $\beta_{\mathrm{H}}-$ Spectrin & \\
\hline$s p c-1[51]$ & a-Spectrin & \\
\hline$h m r-1[35]$ & Cadherin & \\
\hline$h m p-1$ [35] & a-Catenin & \\
\hline hmp-2 [35] & $\beta$-Catenin & \\
\hline$m / c-4[56,62]$ & Nonmuscle myosin light chain & \\
\hline$n m y-1, n m y-2[57]$ & Nonmuscle myosin heavy chain & \\
\hline let-502 $[57,58,59,60]$ & Rho-binding kinase & \\
\hline mel-11 $[57,58,59,60]$ & Myosin phosphatase & \\
\hline fem-2 [60] & PP2c phosphatase & \\
\hline $\operatorname{mig}-2[59,60]$ & Rho/Rac-like GTPase & \\
\hline ced-10 [unpubl. obs.] & Rac GTPase & \\
\hline unc-73 $[59,60]$ & Rho/Rac exchange factor & \\
\hline age-1 [60] & PI3 kinase & \\
\hline daf-2 [60] & Insulin receptor & \\
\hline daf-16 [60] & Forkhead transcription factor & \\
\hline pat $[63,64,65]$ & Muscle or hemidesmosome components & \\
\hline sqt-3 [19] & Collagen & Postelongation \\
\hline
\end{tabular}

neuroblasts. Contralateral leading edge cells meet at the ventral midline and form adherens junctions (AJs, apically located electron dense structures important for linking cells into a single sheet). The ventral surface of the embryo becomes fully enclosed as a purse-string contraction draws the ventral "pocket" cells, which lie posterior to the leading edge cells, to the ventral midline. 

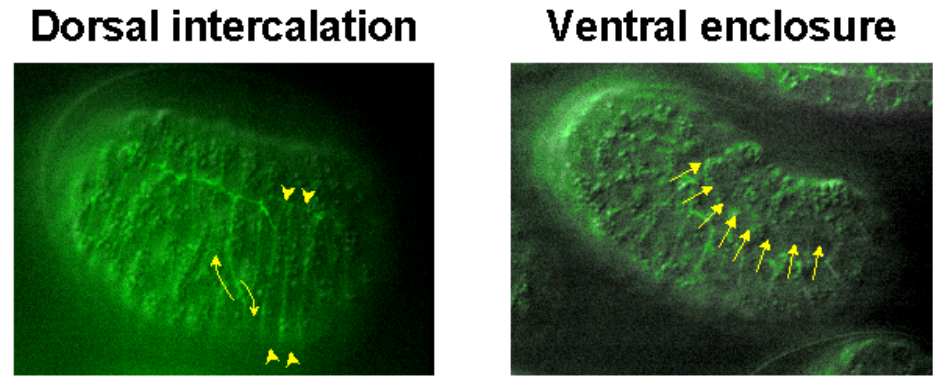

\section{Elongation}
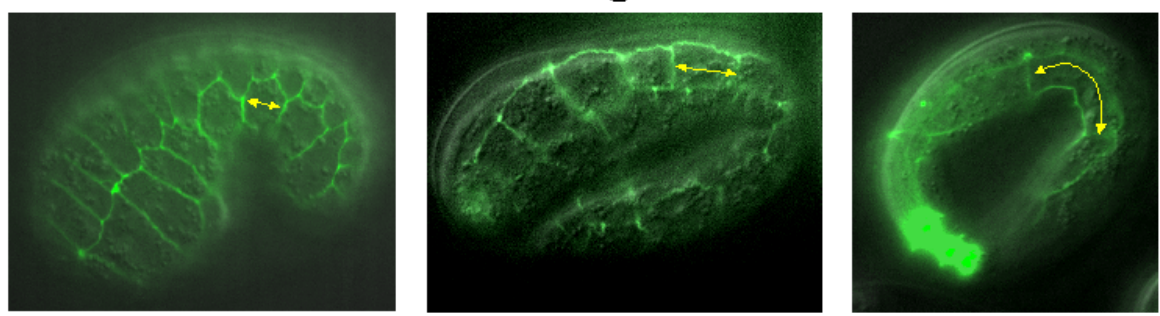

FIGURE 2. Nomarski images of living embryos expressing AJM-1::GFP (green fluorescent protein) at different morphogenetic stages. AJM$1:: G F P$ (green) outlines the epidermal cells. A dorsal/lateral view of an embryo during the latter stages of dorsal intercalation is shown [top left]. A pair of wedge-shaped intercalating dorsal cells are moving past one another as indicated by the arrows. The adjacent pair of fully interdigitated cells are marked by the arrowheads. A lateral view of an embryo undergoing ventral enclosure is shown at the top right. The leading edge ventral epidermal cells are migrating toward the ventral midline as indicated by the arrows. Lateral views of embryos at various stages of elongation are shown in the bottom row. An arrow in a representative lateral epidermal "seam" cell at each stage highlights how these cells are lengthening along the anterior/posterior axis as elongation progresses (the bright anterior GFP signal is the pharynx).

The vab-1 (variable abnormal) and efn (ephrin) genes define the migratory path of the epidermal cells. In anterior regions of vab or efn mutant embryos, the leading edge cells send out abnormal filopodia and migrate slowly or not at all, or migration to the ventral midline occurs but AJ formation fails[25,26,27]. These mutants also have incompletely penetrant defects in the closure of the gastrulation cleft and head and tail morphogenesis. vab-1 and efn 1 through 4 encode homologues of Eph receptor tyrosine kinase and ephrin, respectively, which in other organisms are involved in axon guidance, cell migration, and adhesion[25,26,27,28]. Recent experiments have linked Eph signaling to changes in the cytoskeleton through Rho GTPases[28]. However, vab-1 and efn-1 are both expressed in neuroblasts and function nonautonomously to affect epidermal morphogenesis.

Genetic experiments suggest that efn-4 could function in a pathway with mab-20 (male abnormal), which encodes Semaphorin-2a, although how this would occur still remains to be investigated[27,29]. $m a b-20$ is required to prevent ectopic filopodia formation during ventral enclosure[29]. Normally, only anterior leading edge cells extend filopodia during ventral enclosure[8]. However, in mab-20 mutants the posterior ventral pocket cells also send out actin-rich processes. These cells develop inappropriate contacts that fail to form AJs with their contralateral neighbors causing embryos to rupture at the ventral midline later during elongation[29]. In higher eukaryotes, semaphorins sometimes function as repulsive cues, promoting axon growth cone collapse and repulsion. Recent work has linked the semaphorin Plexin receptors to Rac signaling[30]. Rac and other Rho family GTPases can promote or inhibit filopodia formation[8,9,10,31]. Semaphorin-2a might signal Rac to inhibit filopodia formation in the posterior ventral pocket cells, while still allowing filopodia to form in the more anterior leading edge cells. In addition, ptp-3, which encodes a member of the LAR subfamily of receptor tyrosine phosphatase, displays variable abnormal phenotypes similar to $v a b-1$ and the efns[32]. ptp-3 likely is involved in 
another, partially redundant pathway with $v a b-1$ and $e f n-4 / m a b-20$, and together they ensure the careful coordination of epidermal cell migration over the underlying neuroblasts during ventral enclosure[27].

Consistent with a general role for the Rac system in cell movement, the Arp2/3 complex, gex-2, gex3 , and ced-10 (cell death abnormal, a Rac homolog) display ventral enclosure defects. Epidermal cells either fail to migrate to the ventral midline or fail to form stable contacts causing embryos to rupture and organs, including the gut, are externalized[22,23,33]. Products of all three genes localize to epidermal cell boundaries during wild-type morphogenesis[22,34].

Linkage of the actin cytoskeleton to the membrane is required for formation of stable contacts between contralateral neighbors once they meet at the ventral midline. Classic catenin/cadherins link the actin cytoskeleton to the membrane and are important for ventral enclosure. Cadherins contain extracellular domain(s) for $\mathrm{Ca}^{2+}$-dependent homotypic interactions and intracellular domain(s) that interact with $\beta$-catenin. $\beta$-Catenin in turn links cadherin to $\alpha$-catenin, which directly interacts with actin[35]. In C. elegans, hmp-1, hmp-2, and $h m r-1$ encode $\alpha$-catenin, $\beta$-catenin, and cadherin, respectively[35]. $h m p$ (humpback) mutants display abnormal bulging on their dorsal surface while $h m r$ (hammerhead) fails to fully enclose and bulge at the anterior surface. Although loss of zygotic hmp-1 or $h m p-2$ results in elongation defects (see below), simultaneous loss of both maternal and zygotic hmp- 1 or $h m p-2$ causes ventral enclosure defects similar to those observed in zygotic $h m r-1$ mutants. For these latter mutants, ventral epidermal cells initiate migration correctly, but the anterior leading edge cells may fail to form initial fine contacts with their contralateral neighbors, and the cells never reach the ventral midline. Embryos subsequently rupture during elongation. The products of the three genes localize to AJs when the leading ventral epidermal cells meet at the ventral midline, suggesting that one of the roles of catenin/cadherin is the formation and/or stabilization of contralateral cell-cell contacts required for final enclosure of the embryo.

As described earlier, enclosure is completed when the posterior ventral pocket cells are drawn together by a purse-string contraction similar to those described in Drosophila dorsal closure and wound healing in higher eukaryotes. In Drosophila, leading edge epidermal cells elongate and extend filopodia over the underlying amnioserosa toward the dorsal midline and enclose the embryo after a purse-string contraction draws them together[4,10]. The apparent similarities between Drosophila dorsal closure and C. elegans ventral enclosure may indicate evolutionary conservation of morphogenetic mechanisms. However, while Rho family signaling likely mediates the rearrangement of the actin cytoskeleton in both species, Rho has unique roles in Drosophila that may not be conserved during C. elegans ventral closure. In addition to its effects on actin, Rho also regulates gene expression[8,9,10]. For example, in leading edge cells of the fly, Rho activates the Jun amino-terminal kinase (Jnk) signaling cascade to increase Dpp transcription, and Dpp (a TGF $\beta$-related molecule) in turn signals to neighboring cells, coordinating cell shape changes during dorsal closure[10,36,37,38,39,40]. The C. elegans homologues of Jnk and Dpp pathways apparently are not involved in ventral enclosure[41,42]. Likewise the genes employed for migratory cues in the worm (Eph and ephrin, Semaphorin-2a) play no known role in Drosophila dorsal closure[2,4]. Therefore, only downstream pathways directly regulating the actin cytoskeleton may be conserved between fly dorsal closure and worm ventral enclosure.

\section{ELONGATION}

Following ventral enclosure, dramatic actin-mediated cell shape changes occur in the lateral epidermis, resulting in a fourfold lengthening of the embryo. Priess and Hirsh[19] found that circumferential filamentous bundles of actin (CFBs) form in the lateral epidermal ("seam") cells immediately after ventral enclosure. CFB contraction causes these cells, and the embryo as a whole, to decrease in diameter with a concomitant lengthening of the longitudinal axis (Figs. 2 and 3A)[19]. Treatment of embryos with cytochalasin D disrupts the organization of CFBs and prevents elongation[19]. Many genes are required for elongation and reveal a complex process requiring the coordination of different events and different tissues. 


\section{A Apical view of lateral epidermal cells}
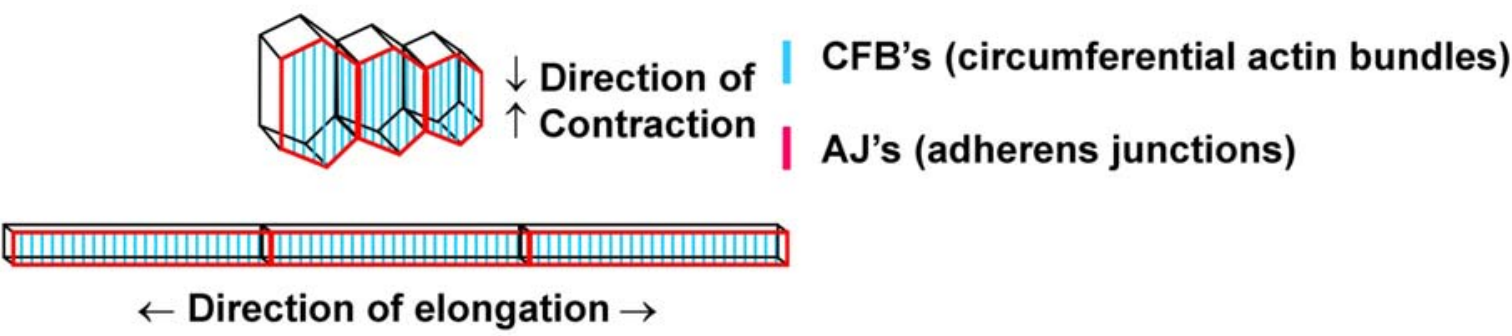

B

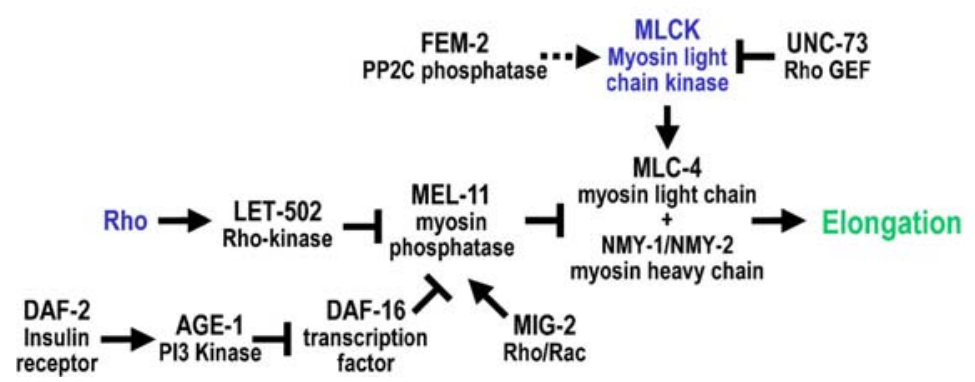

FIGURE 3. (A) A schematic of lateral epidermal "seam" cell shape changes during elongation. The neighboring seam cells are shown at the onset of elongation, and arrows indicate the direction of CFB (in blue) contraction. Seam cells change shape, becoming longer in their longitudinal axis with a concomitant transverse shortening, resulting in a shape change in the direction of the arrows. Neighboring seam cells are linked by AJs (red). (B) The pathway of genes mediating elongation. These interactions were determined by genetic and/or molecular experiments. Positive and negative interactions are indicated by pointed and barred arrows, respectively. Genes not yet shown to interact in the $C$. elegans pathway are colored in blue and are placed based on analogies with other systems. Dashed arrows indicate that the interactions may not be direct.

AJs play a critical role in transmitting the forces of contraction. These structures anchor CFBs to the seam cells' apical surfaces, and other AJs link all of the epidermal cells in a continuous epithelial sheet, allowing the uniform transmission of the contractile force across the embryonic surface[19,43,44]. Microtubules likely act as struts over which the contractile forces stretch the epidermis since disruption of microtubules with nocodazole prevents elongation[19].

let-413 (lethal, a homolog of Drosophila Scribble, an adaptor protein that may interact with small GTPases), $d \underline{\lg -1}$ (disks large, a homolog of Drosophila disks large, a MAGUK membrane-associated guanylate kinase-family scaffolding protein), and ajm-1 (apical junction molecule) all are required for AJ formation, and mutants disrupt elongation[45,46,47,48,49]. LET-413 may define the basolateral border by directing or refining the apical localization of AJ proteins such as DLG-1 and AJM-1[45,46,47,48]. In let413 mutants, DLG-1 and AJM-1 mislocalize to basolateral positions resulting in polarity defects, improper AJ formation, and disorganization of the actin cytoskeleton[49]. Cells of these mutants cannot coordinately change shape, and embryos display elongation defects, often rupturing ventrally. Although ajm-1 and dlg-1 mutant embryos have wild-type actin organization, they fail to elongate, likely because defective AJs result in improper cell association during CFB contraction[45,46,47,48].

sma-1 (small, $\beta_{\mathrm{H}^{-}}$spectrin) and spc-1 ( $\alpha$-spectrin) encode spectrin proteins that anchor CFBs to AJs[50,51]. sma-1 null mutants are viable, but their slowed elongation rate results in short animals[50]. $s p c-1$ mutants are lethal and reach only the point of midelongation (twofold stage, i.e., when the uncurled embryo would be twice the length of the eggshell)[51]. Zygotic loss of hmp-1, hmp-2, and hmr-1 catenin/cadherins result in elongation arrest at stages similar to the spectrins, with the CFBs pulling away from cell membranes[35]. HMP-1, HMP-2, and HMR-1 localize to AJs where they anchor the CFBs (Fig.

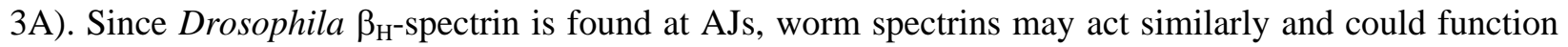
in concert with catenins/cadherins to link the actin cytoskeleton to the membrane[50,51,52]. 
Once the intact epithelium is in place and CFBs are anchored, contraction drives the cell shape changes required for elongation. The actin-based motor complex responsible for CFB contraction is likely thick filaments composed of nonmuscle myosin, which is a hexamer of two heavy chains, two regulatory light chains (rMLC), and two essential light chains[53]. By analogy with smooth muscle contraction and focal adhesion formation in vertebrates, myosin contraction is likely induced by rMLC phosphorylation[8,9,10,53,54]. On activation, myosin light chain kinase (MLCK) or integrin-linked kinase (ILK) phosphorylates rMLC at Ser-19 (Ser-18 in C. elegans) and triggers contraction (Fig. 3B). Myosin phosphatase counteracts MLCK activity by dephosphorylating rMLC, resulting in relaxation. A Rho signaling pathway regulates contraction through its effector, Rho-binding kinase (ROK). At the appropriate time Rho enhances ROK activity, which either phosphorylates myosin phosphatase to render this inhibitor of contraction inactive, and/or directly phosphorylates rMLC. This results in higher levels of phosphorylated rMLC and thus favors contraction[53,54,55].

Mutations in worm homologues of genes in this contractile system indicate that the same pathway operates during $C$. elegans elongation. mlc-4 (rMLC) zygotic null mutants arrest at the twofold stage of elongation due to failed CFB contraction[56]. The two C. elegans nonmuscle myosin genes, nmy-1 and $n m y-2$, act redundantly during elongation and are thus the likely myosin heavy chain partners for $m l c-4$ during embryonic morphogenesis[57].

The C. elegans homologues of ROK (LET-502) and myosin phosphatase (MEL-11) are required for embryonic elongation[57,58,59,60]. let-502 mutants fail to elongate, consistent with ROK/LET-502 inducing CFB contraction. In contrast, mel-11 mutants hyperelongate, likely due to unregulated CFB contraction[58,59,60]. LET-502 is highly expressed within the cytoplasm of elongating epidermal cells and localizes near components of the contractile apparatus. However, MEL-11 is initially cytoplasmic, but becomes sequestered away from the contractile apparatus during elongation in a LET-502-dependent manner[57]. The ROK-dependent localization change of myosin phosphatase also occurs during smooth muscle contraction, likely to prevent inhibition of the contractile machinery[61].

let-502 and mel-11 null mutants suppress one another, indicating that when both functions are removed, embryos elongate almost normally[58,59,60]. If the pathway was linear, one would expect to see the downstream phenotype (mel-11) in the double mutant; instead the double mutants grow to adults, albeit sterile and lumpy/dumpy in appearance. This implies that another pathway functions to regulate elongation in the absence of both let-502 and mel-11; defects are only seen when the two activities are out of balance[60]. We have identified the PP2c phosphatase sex determination gene fem-2 (feminization) as a player in this redundant pathway, although other sex determination genes are apparently not involved in elongation[60]. fem-2 mutants display low penetrance elongation defects; however, triple mutants with let-502 and mel-11 display severe elongation defects, presumably because both the let-502/mel-11 and the parallel fem-2 pathways are inactivated (Fig. 3B).

Several genes that regulate the worm actin cytoskeleton in other processes genetically interact with mel-11 during elongation, although their effects are relatively weak. These genes include mig-2 (Rho/Rac-like), ced-10 (Rac), and unc-73 (Rho/Rac guanine exchange factor)[59,60; P. Mains, unpublished]. Members of the insulin signaling pathway also interact genetically with mel-11; these include age-1 (PI3 kinase), daf-2 (insulin receptor), daf-16 (forkhead transcription factor)[59,60]. Double and triple mutant analysis in combination to analogies with other systems led to the development of the model in Fig. 3B.

LET-502 and MEL-11 appear to function as part of a contractile cassette that is utilized for other contractile events within C. elegans embryos. Maternal LET-502 and MEL-11 localize to cleavage furrows where they regulate the rate of furrow ingression during cytokinesis[62]. As during elongation, mlc-4 encodes the myosin light chain that interacts with LET-502/MEL-11; cytokinesis fails when mlc-4 activity is removed[56], and MLC-4 phosphorylation levels change in the predicted manner in let-502 and mel-11 mutants[62]. Another similarity between cytokinesis and elongation is that let-502 and mel-11 suppress one another in both instances, and the pathways are redundant for both elongation and cytokinesis[62]. However, there are differences between cytokinesis and elongation as the other genes in Fig. 3B play no apparent role in cytokinesis, and genes that genetically interact with let-502 during 
cytokinesis do not affect elongation. Both let-502 and mel-11 are also employed during spermathecal contractions, but they are expressed in different tissues and do not suppress one another[59]. Therefore, the core let-502/mel-11 contractile cassette is used repeatedly throughout development, but accessory proteins differ between processes.

Although its mechanistic link to elongation is unclear, activation of muscle twitching is required for elongation. Longitudinal bands of muscles underlie the epidermis and form contacts, termed hemidesmosomes, with the epidermal basal lamina. Near the beginning of elongation, muscle myofilaments organize and begin twitching. Mutations in genes with a "Pat" phenotype (paralyzed arrest at two-fold) do not twitch and either disrupt muscle formation, function, or hemidesmosomal anchoring to the epidermal cells. For example, pat-4, which encodes ILK, displays a muscle phenotype due to failed hemidesmosome formation[63]. It is not clear why muscle twitching is important for elongation; perhaps nonactive muscle forms a passive mass that impedes elongation or active muscle helps to stretch the expanding epidermis or plays an inductive role with the overlying epidermis[64]. An intriguing possibility is that the muscle attachment structures may induce proper alignment of the CFBs in the epidermal cells[65]. Regardless, both the epidermis and the muscle are essential for elongation; neither alone is sufficient.

\section{POST ELONGATION - MAINTAINING SHAPE}

The cuticle is secreted from the apical surface of the epidermal cells, and this maintains the vermiform shape. Treatment of the embryo with cytochalasin D during elongation causes the embryo to retract in length; however, addition of the drug after elongation is completed has no effect, presumably because the rigid cuticle has locked in the shape[20]. Cuticle-defective mutations in the collagen gene sqt-3 (squat) elongate normally but are unable to maintain their shape[19].

\section{SUMMARY}

C. elegans embryos undergo several morphogenetic events that transform the embryo from a ball of cells into the familiar worm shape. These events involve cell movements to encase the embryo, forcegenerating cell shape changes and formation of an intact epithelial sheet to transmit those forces across the embryonic surface. We are just beginning to understand the regulation of these morphogenetic events, but new genes involved in C. elegans embryonic morphogenesis are continuously being uncovered. Many of the genes regulating the actin cytoskeleton are highly conserved among eukaryotes, including members of the Rho gene family, their regulators, and effectors, and these play critical roles in C. elegans morphogenesis. Since mechanisms analogous to embryonic morphogenesis are utilized elsewhere during C. elegans development and are also employed in other organisms for process like filopodia formation, cell migration, axon outgrowth, and smooth muscle contraction, study of one system will undoubtedly shed light on many others.

\section{REFERENCES}

1. Kiehart, D.P. (1999) Wound healing: the power of the purse string. Curr. Biol. 9, R602-R605.

2. Jacinto, A. and Martin, P. (2001) Morphogenesis: unraveling the cell biology of hole closure. Curr. Biol. 11, R705-R707.

3. Martin-Blanco, E. and Knust, E. (2001) Epithelial morphogenesis: filopodia at work. Curr. Biol. 11, R28-R31.

4. Knust, E. (1997) Drosophila morphogenesis: movements behind the edge. Curr. Biol. 7, R558-R561.

5. Chin-Sang, I.D. and Chisholm, A.D. (2000) Form of the worm: genetics of epidermal morphogenesis in $C$. elegans. Trends Genet. 16, 544-551.

6. Simske, J.S. and Hardin, J. (2001) Getting into shape: epidermal morphogenesis in Caenorhabditis elegans embryos. BioEssays 22, 12-23. 
7. Vasioukhin, V. and Fuchs, E. (2001) Actin dynamics and cell-cell adhesion in epithelia. Curr. Opin. Cell Biol. 13, 76-84.

8. Van Aelst, L. and D’Souza-Schorey, D. (1997) Rho GTPases and signaling networks. Genes Dev. 11, $2295-2322$.

9. $\quad H a l l$, A. (1998) Rho GTPases and the actin cytoskeleton. Science 279, 509-514.

10. Van Aelst, L. and Symons, M. (2002) Role of Rho family GTPases in epithelial morphogenesis. Genes Dev. 16, 1032-1054.

11. Schnabel, R., Hutter, H., Moerman, D., and Schnabel, H. (1997) Assessing normal embryogenesis in Caenorhabditis elegans using a 4D microscope: variability of development and regional specification. Dev. Biol. 184, 234-265.

12. Sulston, J.E., Schierenberg, E., White, J.G., and Thomson, J.N. (1983) The embryonic cell lineage of the nematode Caenorhabditis elegans. Dev. Biol. 100, 64-119.

13. Brenner, S. (1974) The genetics of Caenorhabditis elegans. Genetics 77, 71-94.

14. Riddle, D.L., Blumenthal, T., Meyer, B.J., and Priess, J.R., Eds. (1997) Caenorhabditis elegans II. Cold Spring Harbor Press, Cold Spring Harbor, NY.

15. C. elegans Sequencing Consortium (1998) Genome sequence of the nematode C. elegans: a platform for investigating biology. Science 282, 2012-2018.

16. Fire, A., Xu, S., Montgomery, M.K., Kostas, S.A., Driver, S.E., and Mello, C.C. (1998) Patent and specific genetic interference by double-stranded RNA in Caenorhabditis elegans. Nature 391, 806-811.

17. Williams-Masson, E.M., Heid, P.J., Lavin, C.A., and Hardin, J. (1998) The cellular mechanism of epithelial rearrangement during morphogenesis of the Caenorhabditis elegans dorsal hypodermis. Dev. Biol. 204, $263-276$.

18. Williams-Masson, E.M., Malik, A.N., and Hardin, J. (1997) An actin-mediated two-step mechanism is required for ventral enclosure of the C. elegans hypodermis. Development 124, 2889-2901.

19. Priess, J.R. and Hirsh, D.I. (1986) Caenorhabditis elegans morphogenesis: the role of the cytoskeleton in elongation of the embryo. Dev. Biol. 117, 156-173.

20. Costa, M., Draper, B.W., and Priess, J.R. (1997) The role of actin filaments in patterning the Caenorhabditis elegans cuticle. Dev. Biol. 184, 373-384.

21. Heid, P.J., Raich, W.B., Smith, R., Mohler, W.A., Simokat, K., Gendreau, S.B., Rothman, J.H., and Hardin, J. (2001) The zinc finger protein DIE-1 is required for late events during epithelial cell rearrangement in C. elegans. Dev. Biol. 236, 165-180.

22. Soto, M.C., Qadota, H., Kasuya, K., Inoue, M., Tsuboi, D., Mello, C.C., and Kaibuchi, K. (2002) The GEX-2 and GEX-3 proteins are required for tissue morphogenesis and cell migrations in C. elegans. Genes Dev. 16, 620-632. Severson, A.F., Baillie, D.L., and Bowerman, B. (2002) A formin homology protein and a profilin are required for cytokinesis and Arp2/3-Independent assembly of cortical microfilaments in C. elegans. Curr. Biol. 12, 20662075.

Higgs, H.N. and Pollard, T.D. (2001) Regulation of actin filament network formation through ARP2/3 complex: Activation by a diverse array of proteins. Annu. Rev. Biochem. 70, 649-676.

25. George, S.E., Simokat, K., Hardin, J., and Chisholm, A.D. (1998) The VAB-1 Eph receptor tyrosine kinase functions in neural and epithelial morphogenesis in C. elegans. Cell 92, 633-643.

26. Chin-Sang, I.D., George, S.E., Ding, M., Moseley, S.L., Lynch, A.S., and Chisholm, A.D. (1999) The Ephrin VAB-2/EFN-1 functions in neuronal signaling to regulate epidermal morphogenesis in C. elegans. Cell 99, 781790.

27. Chin-Sang, I.D., Moseley, S.L., Ding, M., Harrington, R.J., George, S.E., and Chisholm, A.D. (2002) The divergent C. elegans ephrin EFN-4 functions in embryonic morphogenesis in a pathway independent of the VAB1 Eph receptor. Development 129, 5499-5510.

28. Schmucker, D. and Zipursky, S.L. (2001) Signaling downstream of Eph receptors and Ephrin ligands. Cell 105, 701-704.

29. Roy, P.J., Zheng, H., Warren, C.E., and Culotti, J.G. (2000) mab-20 encodes Semaphorin-2a and is required to prevent ectopic cell contacts during epidermal morphogenesis in Caenorhabditis elegans. Development 127, 755767.

30. Liu, B.P. and Strittmatter, S.M. (2001) Semaphorin-mediated axonal guidance via Rho-related G proteins. Curr. Opin. Cell Biol. 13, 619-626.

31. Nobes, C.D., Hawkins, P., Stephens, L., and Hall, A. (1995) Activation of the small GTP-binding proteins Rho and Rac by growth factor receptors. J. Cell Sci. 108, 225-233.

32. Harrington, R.J., Gutch, M.J., Hengartner, M.O., Tonks, N.K., and Chisholm, A.D. (2002) The C. elegans LARlike receptor tyrosine kinase phosphatase PTP-3 and the VAB-1 Eph receptor tyrosine kinase have partly redundant functions in morphogenesis. Development 129, 2141-2153.

33. Reddien, P.W. and Horvitz, H.R. (2000) CED-2/CrkII and CED-10/Rac control phagocytosis and cell migrations in Caenorhabditis elegans. Nat. Cell Biol. 2, 131-136.

34. Chen, W., Chen, S., Yap, S.F., and Lim, L. (1996) The Caenorhabditis elegans p21-activated kinase (CePAK) colocalizes with CeRac1 and CDC42Ce at epidermal cell boundaries during embryo elongation. J. Biol. Chem. 271, 26362-26368.

35. Costa, M., Raich, W., Agbunag, C., Leung, B., Hardin, J., and Priess, J.R. (1998) A putative catenin-cadherin 
system mediates morphogenesis of the Caenorhabditis elegans embryo. J. Cell Biol. 141, 297-308.

36. Young, P.E., Richman, A.M., Ketchum, A.S., and Kiehart, D.P. (1993) Morphogenesis in Drosophila requires nonmuscle myosin heavy chain function. Genes Dev. 7, 29-41.

37. Halsell, S.R., Chu, B.I., and Kiehart, D.P. (2000) Genetic analysis demonstrates a direct link between Rho signaling and nonmuscle myosin function during Drosophila morphogenesis. Genetics 155, 1253-1265.

38. Kiehart, D.P., Galbraith, C.G., Edwards, K.A., Rickoll, W.L., and Montague, R.A. (2000) Multiple forces contribute to cell sheet morphogenesis for dorsal closure in Drosophila. J. Cell Biol. 149, 471-490.

39. Mizuno, T., Tsutsui, K., and Nishida, Y. (2002) Drosophila myosin phosphatase and its role in dorsal closure. Development 129, 1215-1223.

40. Noselli, S. and Agnes, F. (1999) Roles of the JNK signaling pathway in Drosophila morphogenesis. Curr. Opin. Genet. Dev. 9, 466-472.

41. Patterson, G.I. and Padgett, R.W. (2000) TGF beta-related pathways. Roles in Caenorhabditis elegans development. Trends Genet. 16, 27-33.

42. Kawasaki, M., Hisamoto, N., Iino, Y., Yamamoto, M., Ninomiya-Tsuji, J., and Matsumoto, K. (1999) A Caenorhabditis elegans JNK signal transduction pathway regulates coordinated movement via type-D GABAergic motor neurons. EMBO J. 18, 3604-3615.

43. Labouesse, M. (1997) Deficiency screen based on the monoclonal antibody MH27 to identify genetic loci required for morphogenesis of the Caenorhabditis elegans embryo. Dev. Dyn. 210, 19-32.

44. Michaux, G., Legouis, R., and Labouesse, M. (2001) Epithelial biology: lessons from Caenorhabditis elegans. Gene 277, 83-100.

45. Legouis, R., Gansmuller, A., Sookhareea, S., Bosher, J.M., Baillie, D.L., and Labouesse, M. (2000) LET-413 is a basolateral protein required for assembly of adherens junctions in Caenorhabditis elegans. Nat. Cell. Biol. 2, 415422.

46. Bossinger, O., Klebes, A., Segbert, C., Theres, C., and Knust, E. (2001) Zonula adherens formation in Caenorhabditis elegans requires dlg-1, the homologue of the Drosophila gene discs large. Dev. Biol. 230, $29-42$.

47. Firestein, B.L. and Rongo, C. (2001) DLG-1 is a MAGUK similar to SAP97 and is required for adherens junction formation. Mol. Biol. Cell 12, 3465-3475.

48. Koppen, M., Simske, J.S., Sims, P.A., Firestein, B.L., Hall, D.H., Radice, A.D., Rongo, C., and Hardin, J.D. (2001) Cooperative regulation of AJM-1 controls junctional integrity in Caenorhabditis elegans epithelia. Nat. Cell. Biol. 3, 983-991.

49. McMahon, L., Legouis, R., Vonesch, J., and Labouesse, M. (2001) Assembly of C. elegans apical junctions involves positioning and compaction by LET-413 and protein aggregation by the MAGUK protein DLG-1. J. Cell Sci. 114, 2265-2277.

50. McKeown, C., Praitis, V., and Austin, J. (1998) sma-1 encodes a $\beta_{\mathrm{H}}$-spectrin homolog required for Caenorhabditis elegans morphogenesis. Development 125, 2087-2098.

51. Norman, K.R. and Moerman, D.G. (2002) Alpha spectrin is essential for morphogenesis and body wall muscle formation in Caenorhabditis elegans. J. Cell Biol. 157, 665-677.

52. Thomas, G.H., Zarnescu, D.C., Juedes, A.E., Bales, M.A., Londergan, A., Korte, C.C., and Kiehart, D.P. (1998) Drosophila $\beta \mathrm{H}$-spectrin is essential for development and contributes to specific cell fates in the eye. Development 125, 2125-2134.

53. Somlyo, A.P. and Somlyo, A.V. (2000) Signal transduction by G-proteins, Rho-kinase and protein phosphatase to smooth muscle and non-muscle myosin II. J. Physiol. 522, 177-185.

54. Deng, J.T., Van Lierop, J.E., Sutherland, C., and Walsh, M.P. (2001) Ca2+-independent smooth muscle contraction. A novel function for integrin-linked kinase. J. Biol. Chem. 276, 16365-16373.

55. Amano M., Ito, M., Kimura, K., Fukata, Y., Chihara, K., Nakano, T., Matsuura, Y., and Kaibuchi, K. (1996) Phosphorylation and activation of myosin by Rho-associated kinase (Rho-Kinase). J. Biol. Chem. 271, 2024620249.

56. Shelton, C.A., Carter, J.C., Ellis, G.C., and Bowerman, B. (1999) The nonmuscle myosin regulatory light chain gene $m l c-4$ is required for cytokinesis, anterior-posterior polarity, and body morphology during Caenorhabditis elegans embryogenesis. J. Cell Biol. 146, 439-451.

57. Piekny, A.J., Johnson, J.L., Cham, G.D., and Mains, P.E. (2003) The Caenorhabditis elegans nonmuscle myosin genes nmy-1 and nmy-2 function as redundant components of the let-502/Rho-binding kinase and mel-11/myosin phosphatase pathway during embryonic morphogenesis. Development 130, 5695-5704.

58. Wissmann, A., Ingles, J., McGhee, J.D., and Mains, P.E. (1997) Caenorhabditis elegans LET-502 is related to Rho-binding kinases and human myotonic dystrophy kinase and interacts genetically with a homolog of the regulatory subunit of smooth muscle myosin phosphatase to affect cell shape. Genes Dev. 11, 409-422.

59. Wissmann, A., Ingles, J., and Mains, P.E. (1999) The Caenorhabditis elegans mel-11 myosin phosphatase regulatory subunit affects tissue contraction in the somatic gonad and the embryonic epidermis and genetically interacts with the Rac signaling pathway. Dev. Biol. 209, 111-127.

60. Piekny, A.J., Wissmann, A., and Mains, P.E. (2000) Embryonic morphogenesis in Caenorhabditis elegans integrates the activity of LET-502 Rho-binding kinase, MEL-11 myosin phosphatase, DAF-2 insulin receptor and FEM-2 PP2c phosphatase. Genetics 156, 1671-1689. 
61. Shin, H.M., Je, H.D., Gallant, C., Tao, T.C., Hartshorne, D.J., Ito, M., and Morgan, K.G. (2002) Differential association and localization of myosin phosphatase subunits during agonist-induced signal transduction in smooth muscle. Circ. Res. 90, 546-553.

62. Piekny, A.J. and Mains, P.E. (2002) Rho-binding kinase (LET-502) and myosin phosphatase (MEL-11) regulate cytokinesis in the early Caenorhabditis elegans embryo. J. Cell Sci. 115, 2271-2282.

63. Mackinnon, A.C., Qadota, H., Norman, K.R., Moerman, D.G., and Williams, B.D. (2002) C. elegans PAT-4/ILK functions as an adaptor protein within integrin adhesion complexes. Curr. Biol. 12, 787-797.

64. Williams, B.D. and Waterston, R.H. (1994) Genes critical for muscle development and function in Caenorhabditis elegans identified through lethal mutations. J. Cell Biol. 124, 475-490.

65. Ding, M., Goncharov, A., Jin, Y., and Chisholm, A.D. (2003) C. elegans ankyrin repeat protein VAB-19 is a component of epidermal attachment structures and is essential for epidermal morphogenesis. Development 130, 5791-5801.

\section{This article should be referenced as follows:}

Piekny, A.J. and Mains, P.E. (2003) Squeezing an egg into a worm: C. elegans embryonic morphogenesis. TheScientificWorldJOURNAL 3, 1370-1381. 

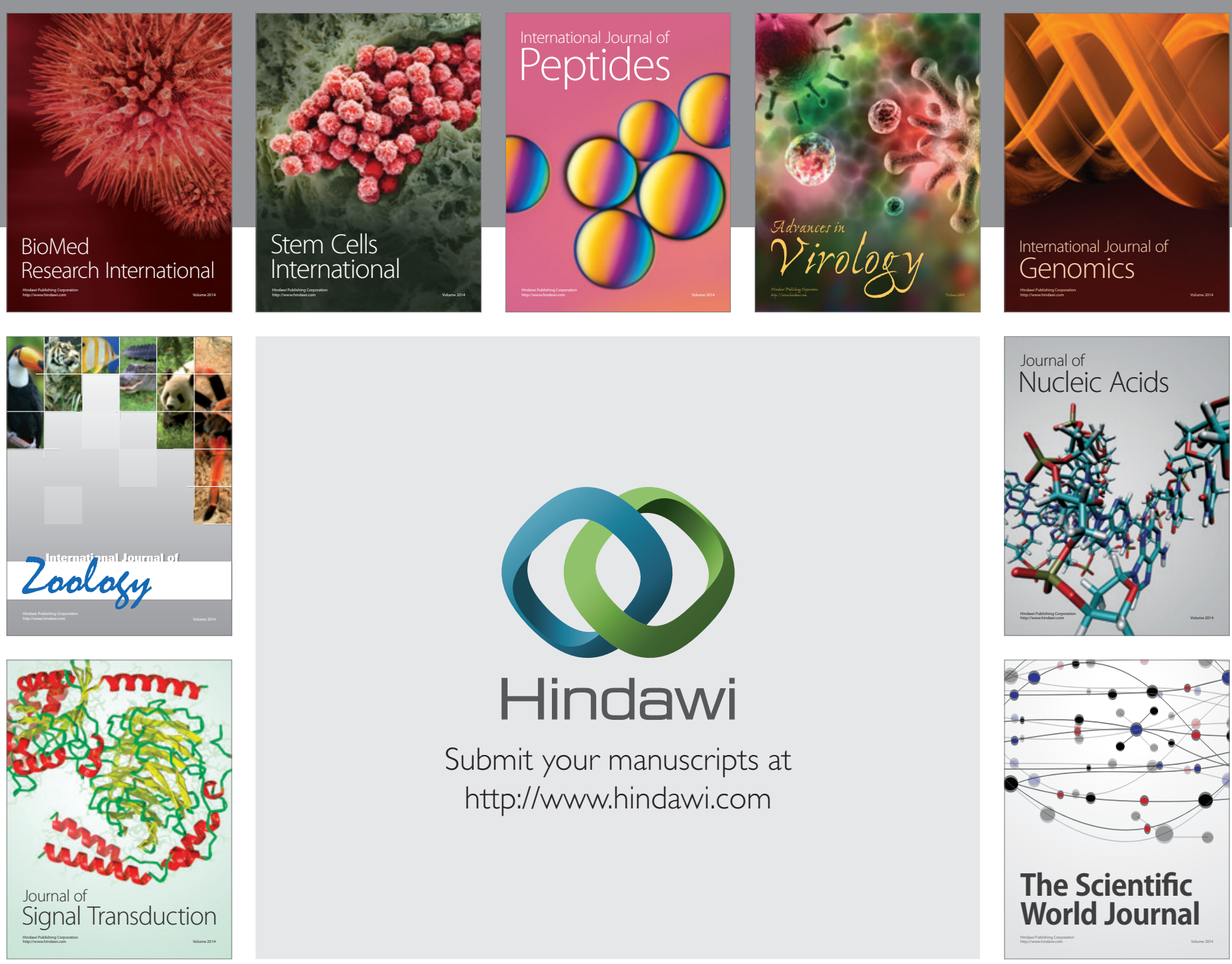

Submit your manuscripts at

http://www.hindawi.com
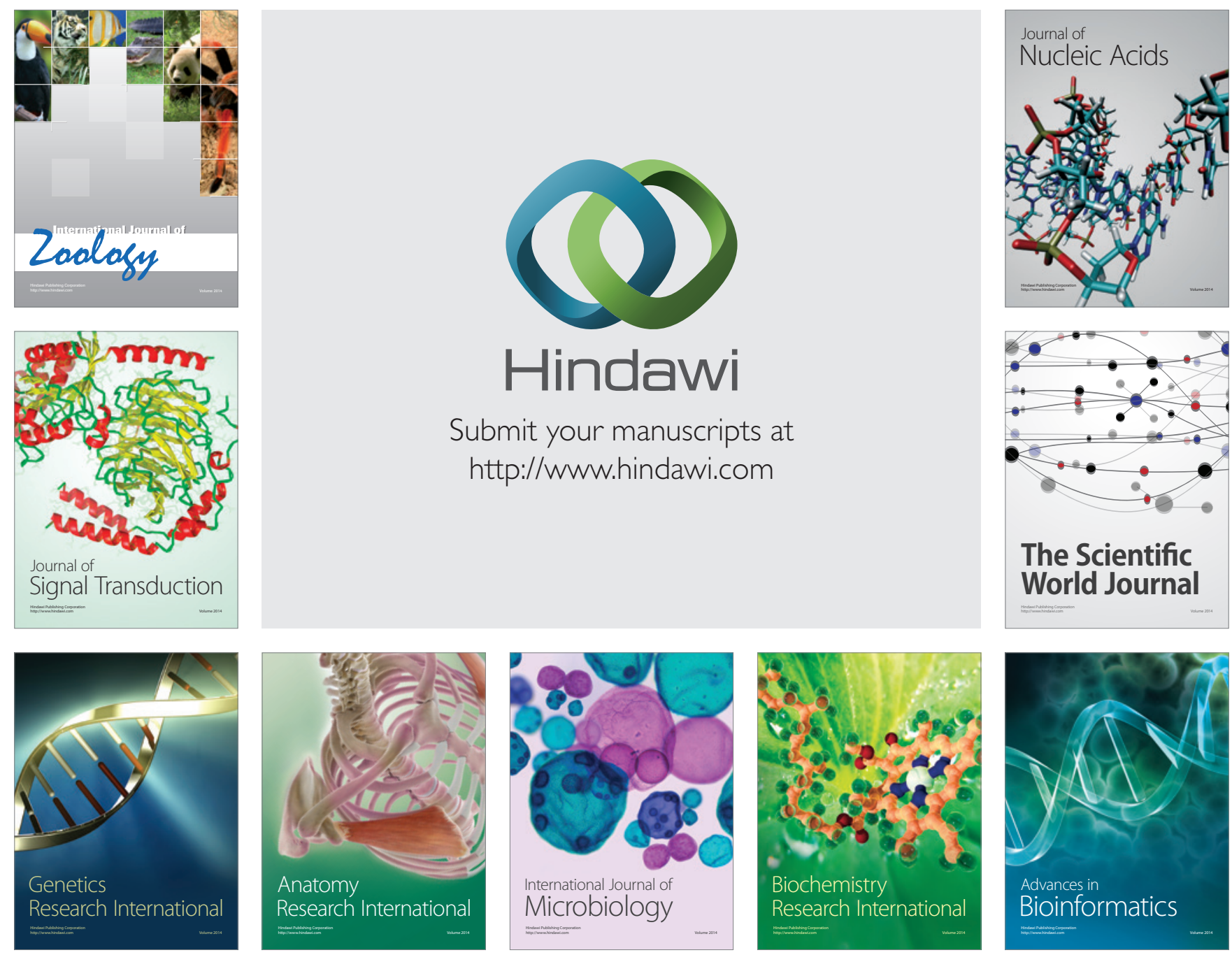

The Scientific World Journal
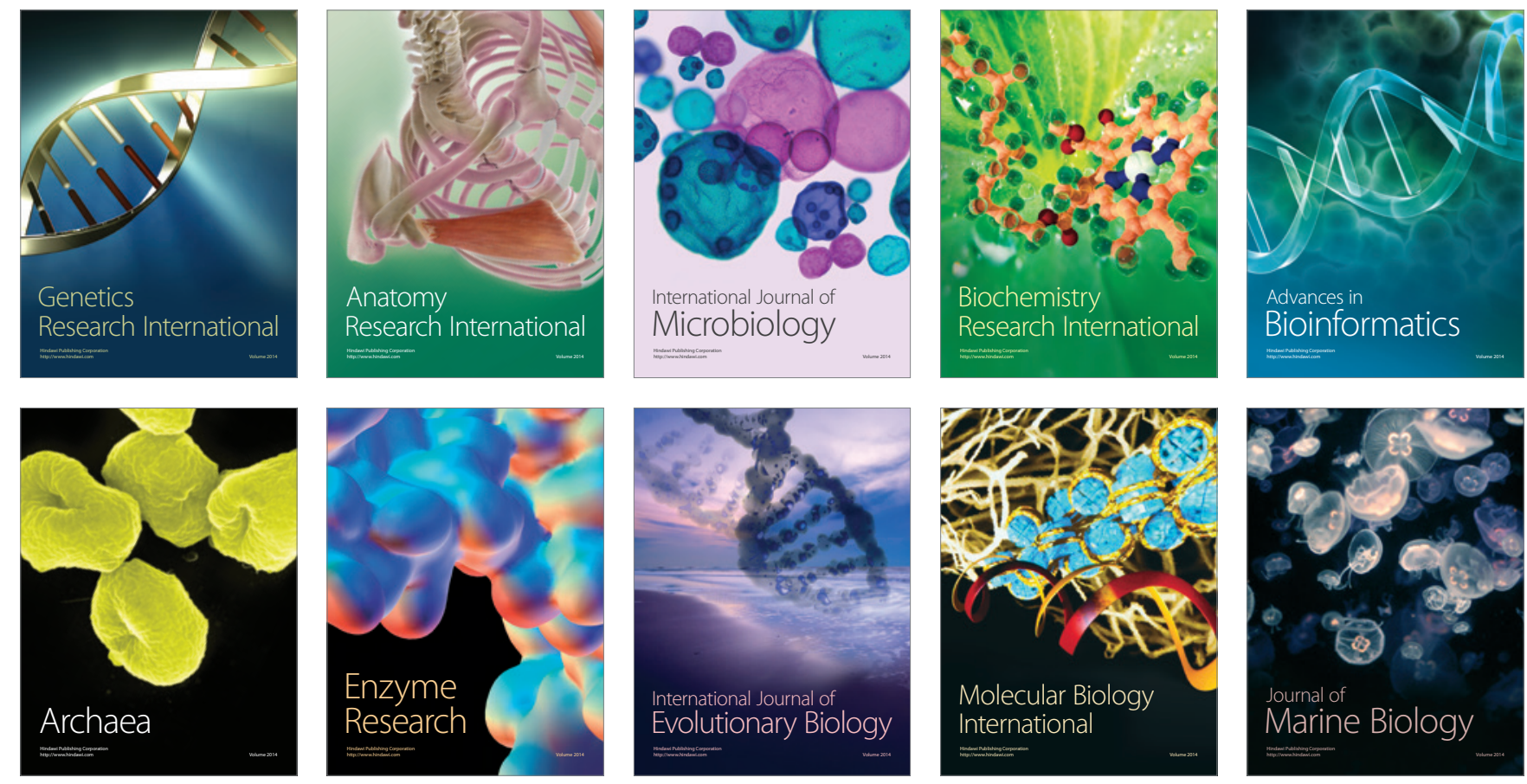